${ }^{1}$ Associate professor, department of Orthodontic and Pediatric Dentistry, College of Dentistry, Qassim University, Qassim, Saudi Arabia.

${ }^{2}$ Associate professor, department of Prosthetic Dental Sciences, College of Dentistry, Qassim University, Qassim, Saudi Arabia.

${ }^{3}$ College of Dentistry, Qassim University, Qassim, Saudi Arabia.

Corresponding author:

Sanaa N. Al-Haj Ali

Department of Orthodontic and Pediatric Dentistry

College of dentistry, Qassim University Qassim, Saudi Arabia

PO Box: 6700 - Postal code: 51452 E-mail:dr.sanaa.alhajali@qudent.org Telephone number: 00966504603339 Fax number: 009660163801762

Author contributions:

R.F, A.A, I.A- research concept and design; A.A, I.A - collection and/ or assembly of data; S. Al. H, R.F data analysis and interpretation; All authors- writing the article; S. Al. $\mathrm{H}$ - critical revision of the article; All authors-final approval of article.

Editor: Dr Altair A. Del Bel Cury

Received: August 28, 2020

Accepted: January 28, 2021

\section{Comparison of Color Difference and Reliability of Two Intraoral Spectrophotometers}

\author{
Sanaa N. Al-Haj Ali ${ }^{1, *}\left(\mathbb{D}\right.$, Ra'fat I. Farah $^{2} \mathbb{D}$, Abdullah \\ Aldhafeeri $^{3}$ (D) Ibrahim Alduraibi ${ }^{3}$ (D)
}

Aim: to evaluate the intra and inter-device reliability of two intraoral spectrophotometers in measuring the Commission Internationale de l'Éclairage (CIE) L*a*b* color coordinates and to compare the color difference $(\Delta \mathrm{E})$ between both devices. Methods: the central region of the labial surface of the maxillary central incisor of 31 participants was measured twice by each of the devices (VITA EasyShade and Degudent Shadepilot) by one examiner. CIE L*a*b* color coordinates were obtained for all teeth and $\triangle E$ was measured and compared. Intraclass correlation coefficient (ICC) and Mann-whitney $U$ test were used to analyze the data $(p<0.05)$. Results: inter-device reliability ICCs in measuring CIE L*a*b* color coordinates ranged between 0.08-0.49 with significant difference between devices only concerning the $b$ coordinate $(p<0.05)$. While intra device reliability ICCs ranged between 0.86-0.89 for VITA EasyShade and 0.81-0.86 for Degudent Shadepilot. The mean $\triangle E$ for $C I E ~ L * a * b *$ color coordinates of VITA EasyShade was $3.61( \pm 1.93)$ compared to $3.60( \pm 1.45)$ for Degudent Shadepilot with insignificant difference between both devices $(p>0.05)$. Conclusions: high intra device reliability in measuring CIE $L^{*} a{ }^{*}{ }^{*}$ color coordinates was achieved particularly of Vita EasyShade, and both devices had clinically acceptable color difference $(\triangle E<3.7)$ however, inter device reliability was low to moderate. Consequently, the same spectrophotometer should be used throughout the steps of performing any tooth- colored restoration.

Keywords: Data accuracy. Collor. Spectrophotometry. 


\section{Introduction}

Correct tooth shade selection is a vital step that significantly affects the esthetic result of tooth- colored restorations, particularly in the anterior region'. Tooth shade selection can be done either visually or by instrumental methods which include spectrophotometers, digital color analyzers, or colorimeters ${ }^{1,2-4}$. Visual shade selection using a dental shade guide which consists of tabs of various shades, despite being popular, is subjective and influenced by several factors which include color deficiency, gender, experience, and eye fatigue, consequently, inconsistencies in shade selection can result which will eventually affect the ability to select the optimum tooth shade ${ }^{5}$.

Spectrophotometers are among the most accurate, useful, and flexible instruments for tooth shade selection ${ }^{6-8}$. Chen et al. ${ }^{8}$ (2012) reported in a systematic review that instrumental color measurements using a spectrophotometer provide the most precise and accurate shade-matching outcomes. Spectrophotometers convert tooth shade into color coordinates as they are operated through the Commission Internationale de l'Éclairage (CIE) L*a*b* color coordinates system (L lightness, a chromaticity along the red-green axis, and b chromaticity along the yellow-blue axis), which allow for an objective shade determination as compared to the visual method $^{6}$. Consequently, they are capable of estimating color by measuring the intensity of reflected light in all visible wavelengths ${ }^{9}$.

Several studies have compared visual shade selection with instrumental shade selection using spectrophotometers and favored spectrophotometers for tooth shade selection ${ }^{8,10-12}$. Among spectrophotometers investigated, VITA EasyShade Advance (VITA Zahnfabrik GmbH, Bad Säckingen, Germany) and Shadepilot (Degudent Shadepilot $\mathrm{GmbH}$, Hanau, Germany) were repeatedly assessed in terms of reliability. Reliability is important as it provides a means of measuring the predictability of the device. A device that produces reliable measurements would likely perform more predictably than a device that is inconsistent ${ }^{13}$. Moderate to high reliability of Vita Easy Shade and Degudent Shadepilot was observed in the studies which assessed these devices, 9 ,13-18 However, different $L{ }^{*}{ }^{\star} b{ }^{\star}$ coordinates were reported to be given by each device ${ }^{17}$.

The objective of this in vivo study was (1) to evaluate the intra-device reliability (agreement of measurements across the same device) and inter-device reliability (agreement of measurements between devices) in measuring CIE $L^{*} a * b *$ color coordinates under clinical conditions of two intraoral spectrophotometers (Vita EasyShade and Degudent Shadepilot), and (2) to compare the color difference $(\triangle \mathrm{E})$ measured from the CIE $L^{\star} a{ }^{*} b^{\star}$ color coordinates between the two devices. The null hypotheses of the current study were that (1) significant agreement exists between the two clinical spectrophotometers, with high intra and inter device reliability and (2) no significant difference in color difference would be present between the two devices.

\section{MATERIAL AND METHODS}

\section{Subjects and ethical approval}

A total of 31 male patients participated in this in vivo study. Ethical approval was obtained from the ethical committee of the college of dentistry/ Qassim University 
before the study was conducted. A written informed consent was also obtained from every participant after a full explanation of the study protocol. The inclusion criteria for the study were:

- Participants in the age range between 20 and 45 years.

- Participants who had intact maxillary right central incisor; free from dental caries, restorations, previous endodontic treatment, and previous bleaching treatment or use of whitening toothpaste.

- Participants must have signed informed written consent.

\section{Study measures}

The tooth color of the upper right central incisor of each of the 31 participants was measured at the dental clinics of the college of dentistry/Qassim University. Shade was recorded for the selected tooth region in each participant by one operator at the central region of its labial surface using VITA EasyShade and Degudent Shadepilot spectrophotometers in two different occasions separated by a one-hour interval. VITA EasyShade and Degudent Shadepilot were selected in this study as they were reported to be among the most reliable spectrophotometers in clinical settings ${ }^{17,19}$. Also, they are both capable of giving different measurements according to the selected tooth region (incisal, central, or cervical). During the measurements, the participants were asked to keep their tongue in a relaxed position away from the selected tooth, lean their head against the headrest of the dental chair, and keep their mouth slightly opened to prevent moving that could affect the measurements. The devices were used and calibrated according to the manufacturer's instructions.

Before measuring the tooth central region color of each participant with the VITA EasyShade, the central incisor was cleaned with a rubber cup and polishing paste for 15 seconds then it was thoroughly washed with water. A disposable infection control shield (VITA Zahnfabrik GmbH, Bad Säckingen, Germany) was used over the face of the probe tip, then the device was calibrated by placing the instrument probe in the calibration block holder such that the probe tip is flush with and perpendicular to the calibration block and depresses the calibration block. Following successful calibration, the central region of the labial surface of the central incisor was measured by placing the instrument probe perpendicular and flush to the tooth surface. Tooth colors expressed in CIE L*a*b* coordinates were obtained and recorded after each measurement. According to manufacturer instructions, measurements with VITA EasyShade were repeated until 2 identical, consecutive measurements of the central region of the tooth were achieved.

On the other hand, measurement with Degudent Shadepilot was preceded by disinfecting its mouthpiece, then the device was calibrated. For measurement, the device was put in the triple-zone measurement mode then the device mouthpiece was put at a right angle to the labial surface of the tooth. Correct positioning was confirmed with the appearance of green horizontal lines. The results were analyzed from the device and the CIE L*a*b* color coordinates of the central region of the teeth were selected and obtained. 


\section{Statistical analysis}

Data were statistically analyzed using the SPSS computer software (Statistical Package for the Social Sciences Version 22, Chicago, IL, USA). Descriptive statistics for the $\mathrm{CIE} L{ }^{*} \mathrm{a} \mathrm{b}^{\star}$ color coordinates obtained from each device were computed, and $\triangle \mathrm{E}$ was calculated using the following equation and compared between both devices:

$$
\Delta \mathrm{E}=1 / 2\left[(\Delta \mathrm{L})^{2}+(\Delta \mathrm{a})^{2}+(\Delta \mathrm{b})^{2}\right]
$$

It has been shown that color difference $(\Delta E)$ between 2.0 and 3.7 was visually detectable under clinical conditions, but it resulted in an acceptable color difference ${ }^{20}$ therefore clinically relevant data in the current study were obtained by counting the number of cases of $\Delta E<2.0$ and $\Delta E<3.7$, respectively.

Because the color differences for each device ( $\triangle E$ values) were not normally distributed, a nonparametric Mann-Whitney $U$ test was used to compare the color difference between both devices. Intra and inter-device reliability of the CIE L*a*b* color coordinates were verified by calculating the Intraclass Correlation Coefficient (ICC). ICC measurements $>0.75$ were employed for reliable measurements. Probability values of $p<0.05$ were considered statistically significant.

\section{RESULTS}

The mean CIE L*a*b* color coordinates $(n=31)$ obtained after measurement of the teeth with VITA Easyshade and Degudent Shadepilot are summarized in Table 1. The mean color difference $(\triangle E)$ for $C I E ~ L * a * b *$ coordinates of each device as well as the medians are summarized in Table 2 . The mean color difference $(\triangle E)$ for $C I E ~ L * a * b *$ color coordinates of VITA Easyshade was $3.61( \pm 1.93)$ while it was $3.60( \pm 1.45)$ for Degudent Shadepilot. Mann-Whitney $U$ test showed that the mean $\triangle E$ for $C I E ~ L * a * b *$ color coordinates between both types of spectrophotometers were significantly indifferent $(p=0.794)$.

Table 1. Mean CIE L*a*b* color coordinates (Standard Deviations) Obtained in 31 participants measured with the VITA EasyShade and Degudent Shadepilot

\begin{tabular}{lccc}
\hline & \multicolumn{1}{c}{$\mathbf{L}^{*}$} & $\mathbf{a}^{*}$ & $\mathbf{b}^{*}$ \\
\hline VITA Easyshade & $77.54(7.83)$ & $-0.177(1.13)$ & $16.37(5.35)$ \\
\hline Degudent Shadepilot & $68.67(12.54)$ & $4.26(2.80)$ & $18.80(6.0)$ \\
\hline
\end{tabular}

Table 2. Means (Standard Deviations) and medians of the calculated $E$ values with both devices with $E$ Cut-off values of 2.0 and 3.7

\begin{tabular}{lccc}
\hline & $\Delta \mathrm{E}($ VITA EasyShade) & $\Delta \mathrm{E}$ (Degudent Shadepilot) & P value \\
\cline { 1 - 3 } Mean (SD) & $3.61(1.93)$ & $3.60(1.45)$ & \multirow{2}{*}{0.794} \\
\cline { 1 - 3 } Median & 3.20 & 3.30 & \\
\cline { 1 - 2 }$<2$ & 19.4 & 9.7 & \\
\hline$\Delta \mathrm{E}<3.7$ & 54.8 & 61.3 & \\
\hline
\end{tabular}

Using Mann-whitney U test 
According to ICC, low inter-device reliability with regards to both $L$ and a color coordinates (ICC: 0.08 and 0.22 respectively) was achieved, while moderate inter-device reliability was noted with regards to the b coordinate (ICC: 0.49) with statistically significant difference $(p=0.02)$ (Table 3). On the other hand, high intra device reliability of the CIE $L{ }^{*} a{ }^{*}{ }^{*}$ color coordinates of both devices was achieved with slightly higher intra device reliability of VITA EasyShade when compared to Degudent Shadepilot (ICC: 0.86-0.89 for VITA Easy Shade compared to 0.81-0.86 for Degudent Shadepilot (Table 4)).

Table 3. Inter-device reliability of color coordinates $\left(L^{*}, a^{*}\right.$ and $\left.b^{*}\right)$

\begin{tabular}{llc}
\hline CIE $L * a * b *$ color coordinate & ICC & -value \\
\hline $\mathrm{L}$ & 0.22 & 0.17 \\
\hline $\mathrm{a}$ & 0.08 & 0.23 \\
\hline $\mathrm{b}$ & 0.49 & $0.02^{*}$ \\
\hline${ }^{*} \mathrm{p}<0.05$ means statistically significant agreement. &
\end{tabular}

${ }^{\star} \mathrm{p}<0.05$ means statistically significant agreement.

Table 4. Intra device reliability of the color coordinates

\begin{tabular}{lcc}
\hline & VITA Easyshade & Degudent Shadepilot \\
\hline$C I E L^{*} \mathrm{a} b *$ Color attribute & ICC & ICC \\
\hline $\mathrm{L}$ & $0.88^{*}$ & $0.82^{*}$ \\
\hline $\mathrm{a}$ & $0.86^{*}$ & $0.86^{*}$ \\
\hline $\mathrm{b}$ & $0.89^{*}$ & $0.81^{*}$ \\
\hline
\end{tabular}

*ICC > 0.75 indicates reliable measurements

\section{DISCUSSION}

In this in vivo study, the CIE L*a*b* color coordinates of the participants' teeth were measured in two different occasions separated by one hour at the central region of the labial surface of the teeth using two commercially available clinical spectrophotometers and both intra device and inter-device reliability of the color coordinates were compared using ICC which is a well-known statistical test for reliability analysis ${ }^{21}$. In addition, the color difference $(\triangle \mathrm{E})$ was calculated in this study for each device and compared. Having such a study in a patient treatment setting instead of a laboratory setting is an advantage as it should represent actual clinical conditions that we face every day more closely ${ }^{7}$. One should consider; however, the complexity involved in clinical instrumental tooth shade selection using a spectrophotometer as compared to in vitro settings as the patient's movement, presence of fogging, the angle and position of the probe, different inclinations, and lightning conditions all can influence the measurements taken and cause misreadings $7,11,19$.

In the current study, the central region of the labial surface of the teeth was chosen as it has been observed that this region lacks the presence of influencing variables which can produce bias in measurements such as the reddish color of adjacent gingiva, the 
greater curvature of the tooth surface in the cervical region, or higher translucency in the incisal region?.

According to the results of the current study, the first null hypothesis is partially rejected as the reported inter-device reliability of the color coordinates was low with regards to both $L$ and a coordinates (ICC: 0.08-0.22) and moderate with regards to the $b$ coordinate (ICC: 0.49 ), which indicates that the color coordinates $(L * a * b)$ given by the two devices varied widely and cannot be exchanged particularly with regards to both $L$ and a coordinates. Having different color coordinates by different spectrophotometers including the ones assessed in the current study was also reported in previous studies $9,14,17,20$. On the other hand, the portion of the null hypothesis which concerns intra device reliability can be accepted as the reported intra device reliability of color coordinates was higher than the inter-device reliability in the current study (ICC value $>0.86$ for VITA EasyShade and $>0.81$ for Degudent Shadepilot) with slightly higher intra device reliability of VITA EasyShade when compared to Degudent Shadepilot. These findings are consistent with the findings of previous studies ${ }^{9,22}$. The resultant low inter-device reliability can be justified by the fact that the color coordinates in CIE L*a*b* color space depend not only on the spectral reflectance curve of an object but also on the light source and the geometrical orientation of the measuring device ${ }^{7,11,19}$. In the absence of a positioning device for both spectrophotometers, it is difficult to ascertain a hundred percent that minor movement while measuring did not occur despite that manufacturers' instructions were strictly followed in both devices as holding the devices steady intraorally is more difficult when compared to having a positioning device ${ }^{13}$.

On the other hand, the slightly lower intra device reliability of the surface measurement device Degudent Shadepilot when compared to the contact type VITA EasyShade can be justified by the fact that the measurements of surface measurement devices are more affected by ambient lighting conditions than those of contact-type devices ${ }^{18}$.

When the mean color difference $(\triangle E)$ of each device is compared to the other in the current study, the result was almost the same with both devices (3.61 with VITA EasyShade vs 3.60 for Degudent Shadepilot) and consequently no significant difference could be found which leads us to accept the second null hypothesis; however, the fact that $45.5 \%$ of the cases with VITA Easy Shade and $38.75 \%$ with Degudent Shadepilot led to an unacceptable color measurement $(\Delta E>3.7)$ can be interpreted as an unsatisfactory outcome. Nevertheless, when that is compared to visual shade selection, where shade matches in only $26.6 \%$ of the cases, this can still be considered acceptable 20 .

Few limitations of the current study should be noted; the first limitation would be that this study compared both devices without having a reference for comparison and comparison with visual shade selection was not also considered. Consequently, the design of the current study does not allow us to know the true color of the participant's teeth. The second limitation would be that this study focused on measuring the central region of the tooth to minimize the bias although in a clinical scenario a reliable spectrophotometer should give reliable measurements for all tooth regions which are prone to be restored. Therefore, future clinical studies should focus on comparing these devices with a reference system and preferably involve a larger number of par- 
ticipants and several teeth and teeth regions. Before conducting these studies, it is recommended that manufacturers train dentists, and even dental technicians, on how to use these devices in ways that can lessen misreadings clinically and improve comparability of readings between different devices.

In conclusion, low to moderate inter-device reliability of Vita Easy Shade and Degudent Shadepilot was achieved in measuring CIE $L{ }^{*} a b^{*}$ color coordinates with a significant difference concerning the $b$ coordinate, while high intra device reliability was achieved particularly of Vita EasyShade. In addition, clinically acceptable mean color difference $(\triangle E<3.7)$ was achieved by both spectrophotometers. Therefore, in a clinical setting and throughout the steps of performing any tooth-colored restoration, which may involve a dental technician, one should consider using the same spectrophotometer; either Vita EasyShade or Degudent Shadepilot for tooth shade selection, without using them interchangeably until manufacturers provide more standardization for the shade selection process.

\section{Conflicts of interest}

The authors declare that they have no conflicts of interest.

\section{Funding statement}

This study received no external funding.

\section{REFERENCES}

1. Bahannan SA. Shade matching quality among dental students using visual and instrumental methods. J Dent. 2014 Jan;42(1):48-52. doi: 10.1016/j.jdent.2013.11.001.

2. Yılmaz B, Irmak Ö, Yaman BC. Outcomes of visual tooth shade selection performed by operators with different experience. J Esthet Restor Dent. 2019 Sep;31(5):500-7. doi: 10.1111/jerd.12507.

3. Farah RI, Elwi H. Spectrophotometric evaluation of color changes of bleach-shade resin-based composites after staining and bleaching. J Contemp Dent Pract. 2014 Sep 1;15(5):587-94. doi: 10.5005/jp-journals-10024-1584.

4. Farah RI. Agreement between digital image analysis and clinical spectrophotometer in $\mathrm{CIEL}{ }^{*} \mathrm{C}^{*} \mathrm{~h}^{\circ}$ coordinate differences and total color difference $(\Delta \mathrm{E})$ measurements of dental ceramic shade tabs. Int J Esthet Dent. 2016;11(2):234-45.

5. Haddad HJ, Jakstat HA, Ametzl G, Borbely J, Vichi A, Dumfahrt H, et al. Does gender and experience influence shade matching quality. J Dent. 2009;37 Suppl 1:e40-4. doi: 10.1016/j.jdent.2009.05.012.

6. Paul SJ, Peter A, Rodoni L, Pietrobon N. Conventional visual vs. spectrophotometric shade taking for porcelain-fused-to-metal crowns: a clinical comparison. Int J Periodontics Restorative Dent. 2004 Jun;24(3):222-31.

7. Witkowski S, Yajima ND, Wolkewitz M, Strub JR. Reliability of shade selection using an intraoral spectrophotometer. Clin Oral Investig. 2012 Jun;16(3):945-9. doi: 10.1007/s00784-011-0590-3.

8. Chen H, Huang J, Dong X, Qian J, He J, Qu X, et al. A systematic review of visual and instrumental measurements for tooth shade matching. Quintessence Int. 2012 Sep;43(8):649-59.

9. Lehmann KM, Devigus A, Igiel C, Wentaschek S, Azar MS, Scheller H. Repeatability of colormeasuring devices. Eur J Esthet Dent. 2011;6(4):428-35. 
10. Kalantari $\mathrm{MH}$, Ghoraishian SA, Mohaghegh M. Evaluation of accuracy of shade selection using two spectrophotometer systems: Vita Easyshade and Degudent Shadepilot. Eur J Dent. 2017 Apr-Jun;11(2):196-200. doi: 10.4103/ejd.ejd_195_16.

11. Igiel $C$, Lehmann $K M$, Ghinea $R$, Weyhrauch $M$, Hangx $Y$, Scheller $H$, et al. Reliability of visual and instrumental color matching. J Esthet Restor Dent. 2017 Sep;29(5):303-8. doi: 10.1111/jerd.12321.

12. Liberato WF, Barreto IC, Costa PP, de Almeida CC, Pimentel W, Tiossi R. A comparison between visual, intraoral scanner, and spectrophotometer shade matching: A clinical study. J Prosthet Dent. 2019 Feb;121(2):271-5. doi: 10.1016/j.prosdent.2018.05.004.

13. Kim-Pusateri S, Brewer JD, Davis EL, Wee AG. Reliability and accuracy of four dental shadematching devices. J Prosthet Dent. 2009 Mar;101(3):193-9. doi: 10.1016/S0022-3913(09)60028-7.

14. Lehmann KM, Igiel C, Schmidtmann I, Scheller H. Four color-measuring devices compared with a spectrophotometric reference system. J Dent. 2010;38 Suppl 2:e65-70. doi: 10.1016/j.jdent.2010.07.006.

15. Gehrke P, Riekeberg U, Fackler O, Dhom G. Comparison of in vivo visual, spectrophotometric and colorimetric shade determination of teeth and implant-supported crowns. Int J Comput Dent. 2009;12(3):247-63.

16. Browning WD, Chan DC, Blalock JS, Brackett MG. A comparison of human raters and an intra-oral spectrophotometer. Oper Dent. 2009;34(3):337-43. doi: 10.2341/08-106.

17. Yuan $\mathrm{K}$, Sun $\mathrm{X}$, Wang $\mathrm{F}$, Wang H, Chen JH. In vitro and in vivo evaluations of three computer-aided shade matching instruments. Oper Dent. 2012;37(3):219-27. doi: 10.2341/11-230-c.

18. Sarafianou A, Kamposiora P, Papavasiliou G, Goula H. Matching repeatability and interdevice agreement of 2 intraoral spectrophotometers. J Prosthet Dent. 2012 Mar;107(3):178-85. doi: 10.1016/S0022-3913(12)60053-5.

19. Dozić A, Kleverlaan CJ, El-Zohairy A, Feilzer AJ, Khashayar G. Performance of five commercially available tooth color-measuring devices. J Prosthodont. 2007;16(2):93-100. doi: 10.1111/j.1532-849X.2007.00163.x.

20. Khashayar G, Dozic A, Kleverlaan CJ, Feilzer AJ. Data comparison between two dental spectrophotometers. Oper Dent. 2012;37(1):12-20. doi: 10.2341/11-161-C.

21. Sabour S, Moezizadeh M, Dastjerdi EV. Reliability of shade selection using an intraoral spectrophotometer: common mistakes in reliability analysis. Clin Oral Investig. 2013 Apr;17(3):1025. doi: 10.1007/s00784-013-0930-6.

22. Lagouvardos PE, Fougia AG, Diamantopoulou SA, Polyzois GL. Repeatability and interdevice reliability of two portable color selection devices in matching and measuring tooth color. $J$ Prosthet Dent. 2009 Jan;101(1):40-5. 